\title{
Traitement chirurgical des pseudarthroses rebelles de l'humérus avec ou sans perte de substance par la technique de la membrane induite
}

\author{
GAILLARD $\mathrm{J}^{1-2}$, MASQUELET AC ${ }^{1}$, BOUTROUX $\mathrm{P}^{1}$, CAMBON-BINDER $\mathrm{A}^{1}$
}

\author{
Auteur correspondant : julien_gaillard94@hotmail.com \\ ${ }^{1}$ Service de Chirurgie Orthopédique et Traumatologique - SOS Main \\ Chirurgie Réparatrice de l'Appareil Locomoteur \\ Hôpital Saint Antoine, 184 rue du Faubourg Saint Antoine, 75012 PARIS, France \\ ${ }^{2}$ Service Chirurgie Orthopédique et Traumatologique \\ Hôpital Américain de Paris \\ 63 Boulevard Victor Hugo, 92200 Neuilly-sur-Seine
}




\section{RESUME}

\section{INTRODUCTION}

Le traitement des pseudarthroses humérales avec ou sans perte de substance osseuse est complexe et comporte un taux de complications et d'échecs non-négligeables. Rares sont les séries qui s'attardent sur la prise en charge des échecs de cure de pseudarthrose humérale.

\section{OBJECTIF}

L'hypothèse de ce travail est que l'utilisation de la membrane induite associée à une ostéosynthèse interne avec deux temps opératoires permet d'obtenir une consolidation de manière constante dans le traitement des pseudarthroses rebelles de l'humérus.

\section{MATERIEL ET METHODES}

L'étude portait sur 15 patients, d'âge médian 46,6 ans. En moyenne, les patients présentaient une pseudarthrose humérale depuis 24 mois et avaient déjà été opérés à 3 reprises pour cure de pseudarthrose. II existait une perte de substance chez 7 patient, elle dépassait $5 \mathrm{~cm}$ dans 2 cas. 6 patients avaient déjà eu une paralysie radiale.

\section{RESULTATS}

La consolidation a été obtenue chez tous les patients. La durée moyenne de consolidation était de 4,6 mois. 10 patients ont eu une transposition du nerf radial, 6 parmi eux ont présenté une paralysie radiale motrice après la transposition. Ils ont tous récupéré entre 2 et 5 mois. 1 cas d'infection superficielle et 1 sérome ont été relevés.

\section{DISCUSSION}

La technique de la membrane induite permet d'obtenir une consolidation osseuse en raison des propriétés biologiques de la membrane. L'inconvénient principal reste celui d'une prise en charge opératoire en 2 temps. Si la perte de substance dépasse $5 \mathrm{~cm}$, l'association d'un segment de fibula multi-perforée et placé à l'intérieur de la membrane augmente la stabilité primaire et participe à l'intégration osseuse

\section{CONCLUSION}

Qu'il y ait ou non une perte de substance, la technique de la membrane induite paraît appropriée au traitement des pseudarthroses de l'humérus. La stratégie en deux interventions reste une mesure de nécessité quand on suspecte une infection larvée. Dans le cadre de cette stratégie en deux temps la transposition antéro-médiale du nerf radial rend plus aisée la deuxième intervention de greffe osseuse.

\section{Etude de niveau IV : Série rétrospective}

MOTS CLES : Pseudarthrose humérale, Membrane induite, Technique de Masquelet, Transposition du nerf radial

KEYWORDS : Humeral non union, Induced membrane, Masquelet Technique, Radial Nerve transposition 


\section{INTRODUCTION}

Les fractures de l'humérus comptent pour 5 à $8 \%$ des fractures [1]. Le taux de pseudarthrose huméral reste fréquent. II varie selon le type de traitement. Tous traitements confondus, le taux de pseudarthrose de l'humérus varie de 0,3 à 15\% [2-4]. En cas de traitement orthopédique d'une fracture humérale, les séries rapportent de $0 \%$ à plus de $30 \%$ de pseudarthroses, alors qu'après prise en charge chirurgicale, les chiffres oscillent entre $4 \%$ et $30 \%[1,5-6]$.

Les pseudarthroses sont généralement caractérisées selon le territoire anatomique concerné, la nature supposée aseptique ou septique, ainsi que sur la présence d'une éventuelle perte de substance.

Plusieurs traitements chirurgicaux peuvent être utilisés pour le traitement des pseudarthroses aseptiques [4,7-8]. Mais peu d'études concernent les pseudarthroses humérales et aucune métanalyse n'a pu être établie [9].

Concernant les pseudarthroses septiques, les séries sont courtes et inhomogènes, confondant différents territoires anatomiques [10]. Seules quelques séries concernent uniquement l'humérus [11-12]. Aucune ne rapporte l'utilisation de la technique de la membrane induite.

Le traitement des pseudarthroses humérales, qu'elles soient avec ou sans perte de substance osseuse est complexe et comporte un taux de complications et d'échecs nonnégligeables. D'une part, la neurolyse itérative du nerf radial peut entrainer un risque de paralysie post opératoire. D'autre part, les échecs de consolidation des cures de pseudarthroses humérales avec fixation interne et greffe osseuse restent élevés. Selon les séries, ils peuvent atteindre 8 à $30 \%$ des cas [2,13-16].

Rares sont les études qui s'attardent sur la prise en charge des échecs de cure de pseudarthrose humérale multi-opérées, souvent appelée " pseudarthroses rebelles » ou " pseudarthroses récalcitrantes ". Quelques solutions ont pu être proposées, sans réelle preuve d'efficacité (BMP7, cellules souches mésenchymateuses, membrane amniotique) [17-19]. Aucune série ne s'attache aux pseudarthroses rebelles de l'humérus.

L'hypothèse de ce travail est que l'utilisation de la membrane induite associée à une ostéosynthèse interne avec deux temps opératoires permet d'obtenir une consolidation de manière constante dans le traitement des pseudarthroses rebelles de l'humérus. 


\section{MATERIEL ET METHODE}

\section{POPULATION (Tableau 1)}

Il s'agit d'une étude rétrospective et monocentrique. Les patients ont été opéré par l'un des 2 chirurgiens seniors référents. Les patients de plus de 18 ans ayant une pseudarthrose humérale traitée par la technique de la membrane induite étaient inclus. II n'y avait pas de critères d'exclusion. 15 patients ont été opérés.

Entre 2014 et 2018, 15 patients ont été opérés pour une pseudarthrose de l'humérus. II s'agissait de 8 femmes et 7 hommes. 13 d'entre eux nous ont été adressés pour prise en charge.

L'âge médian était de 46,6 ans ( $\min 21$ ans - $\max 62$ ans). Quatre patients étaient tabagiques. Trois n'avaient pas arrêté avant la fin de la prise en charge, 1 patient avait arrêté au cours de la prise en charge.

L'atteinte concernait le côté droit dans 8 cas. 4 fractures étaient initialement ouvertes. 6 patients avaient présenté une paralysie radiale pré ou post opératoire lors de l'accident initial. 1 seul en conservait des séquelles motrices définitives. 4 patients présentaient un antécédent documenté d'infection du site opératoire.

La pseudarthrose siégeait beaucoup plus fréquemment au 1/3 moyen - 1/3 inférieur (14 cas) Il existait une perte de substance chez 7 patients, elle dépassait $3 \mathrm{~cm}$ dans 2 cas.

En moyenne, les patients présentaient une pseudarthrose humérale depuis 33.6 mois (min 6 mois - max 144 mois). Le nombre moyen de cure de pseudarthrose préalable était de 3,7 (16). Dix patients avait déjà eu au moins un échec de greffe osseuse iliaque. II s'agissait d'une $1^{\text {ère }}$ cure pour 5 patients. Deux patients présentaient une perte de substance majeure atteignant $8,3 \mathrm{~cm}$ dans un cas et $12,5 \mathrm{~cm}$ pour le second cas. II existait une rupture de matériel pour 4 patients.

\section{TECHNIQUE}

La reconstruction humérale a été réalisée selon la technique de la membrane induite.

Le premier temps consistait en une reprise de l'abord initial (latéral pour 12 cas, postérieur pour 3 cas) avec neurolyse du nerf radial et ablation de tout matériel (Figure 1). La fibrose du foyer de pseudarthrose était réséquée et des prélèvements bactériologiques réalisés, puis le fût médullaire était reperméabilisé.

Pour les 10 derniers patients de la série, une transposition du nerf radial à travers le foyer de pseudarthrose a été réalisée. Après réduction du foyer, une synthèse est réalisée par 1 ou 2 plaque (Figure 2), conditionnées par le siège de la pseudarthrose et le terrain du patient. 
Si la pseudarthrose siégeait en diaphyse humérale, notre choix se portait sur une plaque étroite 4.5/5 (gros fragments). Une seconde plaque 3.5 (petit fragments) a parfois été positionnée dans le but de solidifier le montage, notamment chez les patients obèse, mais au prix d'un encombrement empêchant de laisser la place au future greffon.

En revanche lorsque la pseudarthrose était proche de l'articulation, le positionnement d'une $2^{\text {nde }}$ plaque était bien souvent impossible de par la présence en avant de la fossette olécranienne et en arrière de la fossette coronoidienne.

Les vis à proximité du foyer de pseudarthrose étaient positionnées le plus proche possible du foyer afin de garantir la stabilité du montage et étaient le plus souvent corticales afin de réaliser un montage en compression en absence de perte de substance. Le reste des vis étaient la plupart du temps verrouillées, à l'exception de certaines situations imposées par l'anatomie parfois modifiée par les interventions préalables

La gestion de la perte de substance résiduelle prévisible a été anticipée avant chaque intervention. Celle-ci était évaluée par mesure clinique entre l'humérus sain et l'humérus siège de la pseudarthrose.

Si la perte de substance était jugée inférieure à $3 \mathrm{~cm}$, on réalisait une synthèse en raccourcissement/compression. Puis des lames de ciment sous forme de galette ou de tuile de ciment était disposée en périphérie du foyer en dépassant largement les berges de la pseudarthrose. Dans ces cas où les pseudarthroses étaient ainsi considérées sans perte de substance, 2 à 3 faces de l'os étaient ainsi couvertes par du ciment en engainement de façon à induire une chambre biologique qui manchonne la pseudarthrose.

Si la perte de substance semblait dépasser $3 \mathrm{~cm}$, l'objectif était de s'approcher d'une différence de longueur de $3 \mathrm{~cm}$ maximum en fin d'intervention. La synthèse pontait le defect, un spacer cimenté y était interposé et complété par une entretoise cimentée engainante. Là encore l'intérêt était d'induire une chambre biologique qui manchonne cette fois-ci la perte de substance osseuse.

Le ciment utilisé a été le même pour tous les cas : Ciment Simplex (Stryker). Il s'agit d'un ciment sans antibiotique afin de ne pas masquer une infection larvée lors du $2^{\text {ème }}$ temps opératoire.

Le patient était immobilisé par BABP de façon stricte jusqu'au $2^{\text {ème }}$ temps opératoire avec une contre-écharpe limitant les contraintes en rotation. Les mobilités du poignet et des doigts étaient laissées libres.

Aucune antibiothérapie n'était administrée de façon probabiliste. Une antibiothérapie curative était administrée si les prélèvements bactériologiques étaient positifs.

Le deuxième temps opératoire consiste en l'apport de greffon. Il est classiquement réalisé 6 à 8 semaines après. En cas d'infection diagnostiquée lors du $1^{\mathrm{er}}$ temps opératoire, la seconde intervention était effectuée 15 jours après l'arrêt des antibiotiques. 
L'abord était grandement facilité lorsqu'une transposition du nerf radial avait été réalisée lors de la première intervention avec un nerf radial situé désormais du côté médial du foyer de pseudarthrose et qui restait protégé par la membrane que l'on respecte. La membrane était incisée et les tuiles de ciment ainsi qu'un éventuel spacer étaient enlevés. De nouveaux prélèvements bactériologiques étaient réalisés. Cette membrane ainsi apparente a la forme d'un véritable sac qui comporte deux feuillets, un feuillet consistant en rapport avec les parties molles périphériques et un feuillet plus mince qui tapisse l'os. C'est ce feuillet profond qui est utilisé pour réaliser une véritable décortication " ostéo-membranaire » (Figures 3, 4)

Le temps de décortication est essentiel avant d'apposer le greffon pour stimuler le processus de consolidation. II doit être effectué une décortication ostéo-membranaire à partir de la couche profonde de la membrane sur 2 à $3 \mathrm{~cm}$ de part et d'autre du foyer et de façon la plus circonférentielle possible. Il est impératif de respecter l'enveloppe superficielle de cette membrane. Cette décortication du feuillet profond de l'enveloppe membranaire s'effectue au contact de l'os de sorte que les fragments osseux demeurent pédiculés et vascularisés par la membrane.

Nous n'avons jamais changé le montage lors du $2^{\text {ème }}$ temps opératoire, mais vérifiions systématiquement la bonne tenue des vis.

Nous utilisons systématiquement un apport spongieux autologues qu'il y ait ou non une perte de substance. II est effectué préférentiellement à partir des crêtes iliaques si elles restent disponibles, sinon un RIA nous semble être le meilleur recours (Figure 5-8).

Si la longueur dépasse plus de $5 \mathrm{~cm}$ ( 2 cas), nous avons pris le parti d'associer un segment de fibula non vascularisé mais multi-perforée pour faciliter la revascularisation avec aux extrémités un manchon de greffon iliaque spongieux morcelé (Figures 9,10).

Le patient était ensuite immobilisé de nouveau par BABP de façon stricte pendant 2 mois avec contre écharpe. La rééducation débutait après ce délai concernant le coude et l'épaule.

\section{EVALUATION}

Les patients ont été réévalués respectivement à 1 mois du $1^{\mathrm{er}}$ temps opératoire, puis à 2,4 et 6 mois du $2^{\text {ème }}$ temps opératoires. A 1 an de la prise en charge, un suivi était organisé de façon annuelle.

Un bilan radio clinique était effectué à chaque consultation. La consolidation était évaluée par 2 seniors indépendamment sur des radiographies. La consolidation était retenue lorsque 3 corticales étaient en continuité sur les vues de face et de profil. Un TDM était réalisé entre 4 et 6 mois en cas de doute, notamment chez les patients pour qui la synthèse était effectuée avec 2 plaques pouvant rendant plus difficile l'appréciation d'un cal.

Une éventuelle paralysie radiale était suivie au plan sensitif (Weber) et moteur 


\section{RESULTATS}

La consolidation a été obtenue chez tous les patients.

La durée moyenne de consolidation était de 4,6 mois (min 4 mois - max 9 mois).

La durée moyenne de suivi était de 24 mois (min 6 mois - max 30 mois).

10 patients ont eu une transposition du nerf radial, 6 parmi eux ont présenté une paralysie radiale motrice après la transposition. Ils ont tous récupéré dans un délai entre 2 et 5 mois. Il est intéressant de noter que les 6 paralysies radiales que nous avons constatées en post opératoire concernaient les 6 patients qui avaient déjà eu un antécédent de paralysie radiale.

Il a été retrouvé des germes pour 1 patient au cours de notre prise en charge. Une antibiothérapie était mise en place pour 45 jours et le temps de greffe osseuse décalé après 15 jours d'arrêt des antibiotiques. Aucun germe n'avait été retrouvé lors de la $2{ }^{\text {ème }}$ intervention.

1 cas d'infection superficielle et 1 sérome ont fait l'objet de reprise avec des suites non compliquées. L'infection superficielle a été évacuée chirurgicalement et n'allait pas jusqu'au site opératoire. Une antibiothérapie a été instaurée pour 15 jours.

Aucun patient ne se plaignait de douleurs post opératoires. Les mobilités de coude n'ont pas été modifiées par notre prise en charge 
La tactique opératoire d'une pseudarthrose humérale va dépendre en premier lieu du site anatomique concerné : $1 / 3$ proximal, médio-diaphysaire ou 1/3 distal [16]. Les grands principes thérapeutiques restent identiques mais chaque localisation présente des risques spécifiques[16]. II n'existe à ce jour pas de consensus définissant le meilleur moyen de traiter une pseudarthrose. Les conduites à tenir peuvent différer en cas de d'infection active ou ancienne, ou encore en fonction de la taille du defect osseux. Kontakis a proposé un algorithme de prise en charge des pseudarthroses atrophiques de l'humérus à l'aide de 3 techniques différentes : enclouage centromédullaire + décortication + greffe, vissage par plaque + décortication + greffe, fixation externe selon les principes d'llizarov +/- greffe osseuse [20]. Calori et al. ont proposé un score caractérisant les pseudarthroses des os longs afin d'en guider le traitement [21]. II reste surtout cependant utilisé au membre inférieur et le choix de l'ostéosynthèse n'est pas défini.

A partir d'une revue de la littérature, Peters et al. ont évalué les taux de consolidation après traitement chirurgical des pseudarthroses de la diaphyse humérale en fonction notamment de la synthèse utilisée (plaque, clou, fixateur externe) avec ou sans greffe osseuse [9]. On en retiendra notamment le manque d'efficacité de l'alésage/enclouage centromédullaire. Rares et courtes sont les séries qui s'intéressent au traitement des pseudarthroses humérales septiques [11-12]. Aucune série ne fait état du traitement par membrane induite

Le traitement des pseudarthrose humérales ne fait donc pas consensus. De nombreuses séries existent dans la littérature et sont rapportées dans les tableaux 2, 3 et 4 [22-41]. Les 2 grandes méta analyses de Peters et Kontakis s'accordent à dire que l'immense majorité des articules inclus ont une qualité scientifique relative et qu'il parait difficile d'en déduire une bonne conduite à tenir $[9,20]$.

Ces séries diffèrent entre elles par le mode de synthèse (plaque, enclouage centromédullaire, fixateur externe) et le choix d'apporter ou non une greffe osseuse (Tableaux 2, 3 et 4)

Chaque type de synthèse présente ses avantages et ses inconvénients :

- La plaque contrôlera au mieux les contraintes en distraction/rotation mais nécessite une libération du nerf radial.

- L'enclouage est souvent parfois proposé de nouveau en cas d'enclouage préalable. Effectué après nouveau réalésage, on retient surtout l'idée qu'il soit moins dévascularisant, intérêt qui devient discutable si l'on décide d'aborder le foyer pour adjoindre l'apport osseux. II nous semble cependant dangereux d'effectuer un alésage sans contrôler la position du nerf radial, notamment en cas de perte de substance osseuse.

- Enfin le fixateur externe, proposé nettement moins souvent. Rapporté par certains pour traiter des pseudarthroses atrophiques, il reste pour nous une option intéressante en cas d'infection non contrôlée afin de stabiliser la pseudarthrose sans 
matériel au contact du foyer. Nous pensons cependant qu'un manque de stabilité pourrait être préjudiciable au traitement de la pseudarthrose et préférons relayer le moyen de synthèse par une fixation interne dès que l'infection est contrôlée.

Les pourcentages de consolidation dans ces séries semblent légèrement supérieurs en cas de geste de greffe osseuse associée. Nous associons toujours un geste de greffe osseuse à notre prise en charge et celui-ci nous semble indiscutable en cas de perte de substance osseuse.

Nous avons donc cherché à établir par la technique de la membrane induite une prise en charge systématisée permettant de nous approcher d'un taux de consolidation maximal, qu'il existe ou non une perte de substance associée.

Giannoudis a proposé de démembrer la physiopathologie de la consolidation osseuse. II insiste sur 4 piliers fondamentaux participant à ce processus : la présence de cellules ostéogéniques, une matrice ostéoinductrice, des facteurs de croissance et une stabilité suffisante du foyer de fracture. Il souligne le rôle fondamental de l'apport vasculaire dans la régénération osseuse $[42,43]$. La technique de la membrane induite appliquée aux pseudarthroses de l'humérus permet de participer à ce processus de consolidation osseuse en raison des propriétés biologiques de la membrane. Les études biochimiques réalisées sur la membrane induite démontrent l'existence d'au moins 11 facteurs de croissance (BMP-2, la TGF-Bêta, le VEGF, le Von Willebrand factor, l'interleukine-6, l'Interleukine-8, le collagène type 1, le stroma derived factor-I (STD-1), I'angiotensine-2 (ANG-2), le fibroblast growth factor (FGF-2) et la prostaglandine E2 (PGE-2) [44-48]. Certains auteurs ont ainsi proposé d'adjoindre des facteurs de croissance, avec ou sans greffe osseuse, pour simuler le rôle biochimique de la membrane [49]. On sait également désormais qu'au sein de la membrane se trouvent des celles souches de la lignée mésenchymateuse ainsi que des ostéoblastes [46,50-51]. Cette membrane protège la greffe de la résorption et se comporte comme un promoteur de l'ostéogenèse [52].

L'inconvénient principal reste celui d'une prise en charge opératoire en 2 temps. Cette stratégie nous paraît cependant nécessaire en cas d'ATCD d'infection, idéale en cas de perte de substance, mais également dans les cas de PSA sans perte de substance, la membrane induite formant dans ces situations une virole permettant d'apporter une contention et une protection trophique de la greffe. Avec ou sans perte de substance, la mise en place du ciment doit comprendre un engainement du foyer de pseudarthrose par des galettes dépassant les berges. La membrane induite se développe ainsi telle une virole autour du foyer. Le greffon spongieux pourra être ainsi apposé dans la membrane induite en manchon (Figures 3,4, 6-8).

La transposition du nerf radial a été décrite par Schnitker en 1949 [53]. Des études cadavériques n'ont pas retrouvé d'augmentation de longueur donc de tension [54-55]. Certains l'ont proposé d'emblée lors de la prise en charge de fractures humérales, possiblement en prévision d'un geste futur de reprise [56-59]. Ces neurolyses itératives auxquelles nous avons été confrontées nous ont fait opté pour une transposition 
systématique du nerf lors du $1^{\mathrm{er}}$ temps opératoire. L'éloignement du nerf facilite la mise en place de l'ostéosynthèse et du ciment lors du $1^{\mathrm{er}}$ temps. Le nerf reste protégé en situation rétro-membranaire au cours du $2^{\text {ème }}$ temps.

Notre étude comporte plusieurs faiblesses, à commencer par sa nature rétrospective. L'objectif de ce travail ayant été d'évaluer la consolidation des pseudarthroses par la technique de la membrane induite, nous n'avons pas effectué d'analyse par scores fonctionnels (QuickDASH, Constant, ASES).

La définition du caractère récalcitrant d'une pseudarthrose n'est pas clairement définie (à partir de combien d'échecs chirurgicaux peut-on qualifier une pseudarthrose de récalcitrante ?). Enfin la décision de transposition antérieure du nerf radial n'ayant été systématique qu'après la prise en charge de 5 patients, il peut sembler difficile d'apprécier les conséquences de cette procédure dont l'intérêt se doit d'être démontré. 


\section{CONCLUSION}

Qu'il y ait donc ou non une perte de substance, la technique de la membrane induite nous paraît appropriée au traitement des pseudarthroses rebelles de l'humérus.

La consolidation n'a pour le moment jamais fait défaut. La stratégie en deux interventions, même si elle peut apparaître comme un inconvénient, reste une mesure de nécessité quand on suspecte une infection larvée, ce qui n'est jamais à exclure dans les défauts de consolidation. Dans le cadre de cette stratégie en deux temps la transposition antéromédiale du nerf radial évite la mise en tension du nerf et rend plus aisée la deuxième intervention de greffe osseuse. 


\section{BIBLIOGRAPHIE}

1. Volgas DA, Stannard JP, Alonso JE. Nonunions of the Humerus: Clin Orthop. 2004 Feb;419:46-50.

2. Healy WL, White GM, Mick CA, Brooker AF, Weiland AJ. Nonunion of the humeral shaft. Clin Orthop. 1987 Jun;(219):206-13.

3. Heim D, Herkert F, Hess P, Regazzoni P. Surgical treatment of humeral shaft fractures--the Basel experience. J Trauma. 1993 Aug;35(2):226-32.

4. Marti RK, Verheyen CCPM, Besselaar PP. Humeral shaft nonunion: evaluation of uniform surgical repair in fifty-one patients. J Orthop Trauma. 2002 Feb;16(2):108-15.

5. Harkin FE, Large RJ. Humeral shaft fractures: union outcomes in a large cohort. J Shoulder Elbow Surg. 2017 Nov;26(11):1881-8.

6. Muramatsu K, Doi K, Ihara K, Shigetomi M, Kawai S. Recalcitrant posttraumatic nonunion of the humerus: 23 patients reconstructed with vascularized bone graft. Acta Orthop Scand. 2003 Jan;74(1):95-7.

7. Singh AK, Arun GR, Narsaria N, Srivastava A. Treatment of non-union of humerus diaphyseal fractures: a prospective study comparing interlocking nail and locking compression plate. Arch Orthop Trauma Surg. 2014 Jul;134(7):947-53.

8. Lin J, Chiang H, Hou S-M. Open exchange locked nailing in humeral nonunions after intramedullary nailing. Clin Orthop. 2003 Jun;(411):260-8.

9. Peters RM, Claessen FMAP, Doornberg JN, Kolovich GP, Diercks RL, van den Bekerom MPJ. Union rate after operative treatment of humeral shaft nonunion - A systematic review. Injury. 2015 Dec;46(12):2314-24.

10. Struijs PAA, Poolman RW, Bhandari M. Infected nonunion of the long bones. J Orthop Trauma. 2007 Aug;21(7):507-11.

11. Ferreira N, Marais LC, Serfontein C. Two stage reconstruction of septic non-union of the humerus with the use of circular external fixation. Injury. 2016 Aug;47(8):1713-8.

12. Liu T, Zhang X, Li Z, Zeng W, Peng D, Sun C. Callus distraction for humeral nonunion with bone loss and limb shortening caused by chronic osteomyelitis. J Bone Joint Surg Br. 2008 Jun;90(6):795800.

13. Haidukewych GJ, Sperling JW. Results of treatment of infected humeral nonunions: the Mayo Clinic experience. Clin Orthop. 2003 Sep;(414):25-30.

14. Beredjiklian PK, Hotchkiss RN, Athanasian EA, Ramsey ML, Katz MA. Recalcitrant nonunion of the distal humerus: treatment with free vascularized bone grafting. Clin Orthop. 2005 Jun;(435):1349.

15. Wood MB. Free vascularized bone transfers for nonunions, segmental gaps, and following tumor resection. Orthopedics. 1986 Jun;9(6):810-6. 
16. King AR, Moran SL, Steinmann SP. Humeral Nonunion. Hand Clin. 2007 Nov;23(4):449-56.

17. Gindraux F, Rondot T, de Billy B, Zwetyenga N, Fricain J-C, Pagnon A, et al. Similarities between induced membrane and amniotic membrane: Novelty for bone repair. Placenta. 2017 Nov 1;59:116-23.

18. Gindraux F, Obert L, Laganier L, Barnouin L. Industrial approach in developing an advanced therapy product for bone repair. J Tissue Eng Regen Med. 2010 Mar 1;4(3):194-204.

19. Obert L, Couesmes A, Petite H, Lepage D, Bonin N, Tropet Y, et al. 83 Pseudarthroses résistante des os longs et BMP 7 : évaluation de la consolidation osseuse radiographique et histologique dans des cas appariés ou compassionnels. Rev Chir Orthop. 2007 Nov;93(7):67-68

20. Kontakis GM, Tosounidis T, Pagkalos J. Humeral diaphyseal aseptic non-unions: an algorithm of management. Injury. 2007;38:S39-49.

21. Calori GM, Colombo M, Mazza EL, Mazzola S, Malagoli E, Marelli N, et al. Validation of the Non-Union Scoring System in 300 long bone non-unions. Injury. 2014 Dec;45 Suppl 6:S93-7.

22. Abalo A, Dosseh ED, Adabra K, Walla A, James YE, Dossim A. Open reduction and internal fixation of humeral non-unions: radiological and functional results. Acta Orthop Belg. 2011;77(3):299- 303.

23. Barquet A, Fernandez A, Luvizio J, Masliah R. A combined therapeutic protocol for aseptic nonunion of the humeral shaft: a report of 25 cases. J Trauma. 1989;29(1):95- 8.

24. Bernard de Dompsure R, Peter R, Hoffmeyer P. Uninfected nonunion of the humeral diaphyses: review of 21 patients treated with shingling, compression plate, and autologous bone graft. Orthop Traumatol Surg Res. 2010;96(2):139- 46.

25. Hierholzer C, Sama D, Toro JB, Peterson M, Helfet DL. Plate fixation of ununited humeral shaft fractures: effect of type of bone graft on healing. J Bone Joint Surg Am. 2006;88(7):1442- 7.

26. Hsu T-L, Chiu F-Y, Chen C-M, Chen T-H. Treatment of nonunion of humeral shaft fracture with dynamic compression plate and cancellous bone graft. J Chin Med Assoc. 2005;68(2):73- 6.

27. Kumar A, Sadiq SA. Non-union of the humeral shaft treated by internal fixation. Int Orthop. 2002;26(4):214- 6.

28. Lin C-L, Fang C-K, Chiu F-Y, Chen C-M, Chen T-H. Revision with dynamic compression plate and cancellous bone graft for aseptic nonunion after surgical treatment of humeral shaft fracture. J Trauma. 2009;67(6):1393- 6.

29. Livani B, Belangero W, Medina G, Pimenta C, Zogaib R, Mongon M. Anterior plating as a surgical alternative in the treatment of humeral shaft non-union. Int Orthop. 2010;34(7):1025- 31.

30. Wang S-H, Chang J-H, Pan R-Y, Chao K-H, Lin L-C, Wang S-J. Treatment of Osteopenic or Nonunited Fractures of the Humerus with Structure Bone Allograft and Non-locking Plates. J Med Sci. 2013;33(2):67- 74. 
31. Dujardin FH, Mazirt N, Tobenas AC, Duparc F, Thomine JM. [Failure of locked centromedullary nailing in pseudarthrosis of the humeral diaphysis]. Rev Chir Orthop Reparatrice Appar Mot. 2000;86(8):773- 80.

32. Flinkkilä T, Ristiniemi J, Hämäläinen M. Nonunion after intramedullary nailing of humeral shaft fractures. J Trauma. 2001;50(3):540- 4.

33. Ilyas I, Younge DA. Locked intramedullary nailing for difficult nonunions of the humeral diaphysis. Int Orthop. 2003;27(5):278- 81.

34. Li X, Wang H, Wei Y, Wu Z. Treatment of nonunions of humeral fractures with interlocking intramedullary nailing. Chin J Traumatol Zhonghua Chuang Shang Za Zhi. 2008;11(6):335- 40.

35. Martínez AA, Herrera A, Cuenca J. Marchetti nailing with decortication and bone graft in nonunions of the two upper thirds of the humerus. Chir Main. 2002;21(1):28- 32.

36. Verbruggen JP a. M, Stapert JWJL. Failure of reamed nailing in humeral non-union: an analysis of 26 patients. Injury. 2005;36(3):430- 8.

37. Bari MM, Ahmed MU, Hossain MB, Chowdhury FR, Siddiqui MA. Treatment of non union of humerus using G. A. Ilizarov technique. Mymensingh Med J. 2004;13(1):36- 8.

38. Kiran M, Jee R. Ilizarov's method for treatment of nonunion of diaphyseal fractures of the humerus. Indian J Orthop. 2010;44(4):444- 7.

39. Lammens J, Bauduin G, Driesen R, Moens P, Stuyck J, De Smet L, et al. Treatment of nonunion of the humerus using the llizarov external fixator. Clin Orthop. 1998;(353):223- 30.

40. Lavini F, Renzi Brivio L, Pizzoli A, Giotakis N, Bartolozzi P. Treatment of non-union of the humerus using the Orthofix external fixator. Injury. 2001;32 Suppl 4:SD35-40.

41. Patel VR, Menon DK, Pool RD, Simonis RB. Nonunion of the humerus after failure of surgical treatment. Management using the llizarov circular fixator. J Bone Joint Surg Br. 2000;82(7):977- 83.

42. Giannoudis PV, Einhorn TA, Marsh D. Fracture healing: the diamond concept. Injury. 2007;38:S3-6.

43. Giannoudis PV, Harwood PJ, Tosounidis T, Kanakaris KN. Restoration of long bone defects treated with the induced membrane technique: protocol and outcomes. Injury 2016 Dec; 47S6:S53S61

44. Pelissier P, Masquelet AC, Bareille R, Pelissier SM, Amedee J. Induced membranes secrete growth factors including vascular and osteoinductive factors and could stimulate bone regeneration. J Orthop Res Off Publ Orthop Res Soc. 2004 Jan;22(1):73-9.

45. Aho O-M, Lehenkari P, Ristiniemi J, Lehtonen S, Risteli J, Leskelä H-V. The mechanism of action of induced membranes in bone repair. J Bone Joint Surg Am. 2013 Apr 3;95(7):597-604.

46. Cuthbert RJ, Churchman SM, Tan HB, McGonagle D, Jones E, Giannoudis PV. Induced periosteum a complex cellular scaffold for the treatment of large bone defects. Bone. 2013 Dec;57(2):484-92. 
47. Christou C, Oliver RA, Yu Y, Walsh WR. The Masquelet technique for membrane induction and the healing of ovine critical sized segmental defects. PloS One. 2014;9(12):e114122.

48. Wang X, Wei F, Luo F, Huang K, Xie Z. Induction of granulation tissue for the secretion of growth factors and the promotion of bone defect repair. J Orthop Surg. 2015 Sep 17;10:147.

49. Miska M, Findeisen S, Tanner M, Biglari B, Studier-Fischer S, Grützner PA, et al. Treatment of nonunions in fractures of the humeral shaft according to the Diamond Concept. Bone Joint J. 2016 Jan;98-B(1):81-7

50. Henrich D, Seebach C, Nau C, Basan S, Relja B, Wilhelm K, et al. Establishment and characterization of the Masquelet induced membrane technique in a rat femur critical-sized defect model. J Tissue Eng Regen Med. 2016;10(10):E382-96.

51. Gouron R, Petit L, Boudot C, Six I, Brazier M, Kamel S, et al. Osteoclasts and their precursors are present in the induced-membrane during bone reconstruction using the Masquelet technique. J Tissue Eng Regen Med. 2017;11(2):382-9.

52. Viateau V, Bensidhoum M, Guillemin G, Petite H, Hannouche D, Anagnostou F, et al. Use of the induced membrane technique for bone tissue engineering purposes: animal studies. Orthop Clin North Am. 2010 Jan;41(1):49-56; table of contents.

53. Schnitker MT. A Technique for Transplant of the Musculospiral Nerve in Open Reduction of Fractures of the Mid-Shaft of the Humerus. J Neurosurg. 1949;6(2):113-7.

54. Yakkanti MR, Roberts CS, Murphy J, Acland RD. Anterior transposition of the radial nerve-a cadaveric study. J Orthop Trauma. 2008;22(10):705-8.

55. Bacakoglu AK, Kiray A, Muratli K, Ekin A, Ergur I. Medial transposition of the radial nerve for anterolateral plate fixation of the humerus: Cadaveric study. Anat Sci Int. 2007 Jun;82(2):116-20.

56. Muzykewicz DA, Abrams RA. Transhumeral Anterior Radial Nerve Transposition to Simplify Anticipated Future Humeral Reconstruction. J Hand Surg. 2017 Jul;42(7):578.e1-578.e5.

57. Olarte CM, Darowish M, Ziran BH. Radial Nerve Transposition With Humeral Fracture Fixation: Preliminary Results. Clin Orthop. 2003 Aug;413:170-4.

58. Chamseddine AH, Abdallah A, Zein $\mathrm{H}$, Taha A. Transfracture medial transposition of the radial nerve associated with plate fixation of the humerus. Int Orthop. 2017 Jul;41(7):1463-70.

59. Chamseddine AH, Zein HK, Alasiry AA, Mansour NA, Bazzal AM. Trans-fracture transposition of the radial nerve during the open approach of humeral shaft fractures. Eur J Orthop Surg Traumatol. 2013 Aug;23(6):725-30. 


\section{TABLEAUX}

\begin{tabular}{|c|c|c|c|c|c|c|c|c|c|c|c|c|c|}
\hline Patients & Age & Sexe & Côté & Tabagisme & $\begin{array}{l}\text { Mécanisme } \\
\text { de la } \\
\text { fracture }\end{array}$ & Localisation & $\begin{array}{l}\text { Ouverture } \\
\text { cutanée } \\
\text { (Gustillo) }\end{array}$ & PR initiale & $\begin{array}{l}\text { Traitement } \\
\text { initial }\end{array}$ & $\begin{array}{c}\text { Nombre } \\
\text { de } \\
\text { chirurgies }\end{array}$ & $\begin{array}{l}\text { PDS } \\
(\mathrm{cm})\end{array}$ & $\begin{array}{c}\text { ATCD } \\
\text { Infectieux }\end{array}$ & $\begin{array}{c}\text { Durée } \\
\text { de la } \\
\text { PSA } \\
\text { (mois) }\end{array}$ \\
\hline 1 & 46 & $\mathrm{~F}$ & $\mathrm{D}$ & 0 & $\begin{array}{c}\text { Radique } \\
\text { (fr. patho) }\end{array}$ & $1 / 3$ moyen & Non & 0 & Orthopédique & 2 & 4 & Non & 12 \\
\hline 2 & 44 & $\mathrm{M}$ & G & 0 & Chute & $\begin{array}{c}1 / 3 \\
\text { inférieur }\end{array}$ & Non & 0 & 1 plaque & 3 & 2 & Non & 18 \\
\hline 3 & 36 & $\mathrm{M}$ & G & 0 & AVP & $1 / 3$ moyen & $\begin{array}{c}\text { Oui } \\
1\end{array}$ & 0 & 1 plaque & 4 & 4 & Oui & 24 \\
\hline 4 & 58 & $\mathrm{~F}$ & G & 0 & AVP & $\begin{array}{c}1 / 3 \\
\text { supérieur }\end{array}$ & Non & 0 & Clou & 3 & 0 & Non & 22 \\
\hline 5 & 50 & $\mathrm{M}$ & $\mathrm{D}$ & 0 & Chute & $\begin{array}{c}1 / 3 \\
\text { inférieur }\end{array}$ & $\begin{array}{c}\text { Oui } \\
2\end{array}$ & $\begin{array}{c}\text { Oui } \\
\text { Récupération } \\
\text { incomplète }\end{array}$ & 2 plaques & 6 & 0 & Oui & 53 \\
\hline 6 & 49 & $\mathrm{~F}$ & D & 0 & AVP & $\begin{array}{c}1 / 3 \\
\text { inférieur }\end{array}$ & $\begin{array}{c}\text { Oui } \\
2 \\
\end{array}$ & 0 & Orthopédique & 6 & 8,3 & Oui & 14 \\
\hline 7 & 21 & $M$ & D & Oui & AVP & $1 / 3$ moyen & $\begin{array}{l}\text { Oui } \\
3 \mathrm{~A}\end{array}$ & $\begin{array}{c}\text { Oui } \\
\text { Récupération } \\
\text { complète }\end{array}$ & 1 plaque & 3 & 0 & Non & 42 \\
\hline 8 & 25 & $\mathrm{~F}$ & G & 0 & Chute & $\begin{array}{c}1 / 3 \\
\text { inférieur }\end{array}$ & Non & 0 & Broches & 3 & 0 & Non & 17 \\
\hline 9 & 56 & $\mathrm{~F}$ & G & 0 & Chute & $\begin{array}{c}1 / 3 \\
\text { inférieur }\end{array}$ & Non & $\begin{array}{c}\text { Oui } \\
\text { Récupération } \\
\text { complète }\end{array}$ & 1 plaque & 3 & 0 & Non & 34 \\
\hline 10 & 48 & $\mathrm{~F}$ & D & Oui & AVP & $1 / 3$ moyen & Non & $\begin{array}{c}\text { Oui } \\
\text { Récupération } \\
\text { complète }\end{array}$ & Clou & 6 & 12,5 & Oui & 144 \\
\hline 11 & 61 & $\mathrm{~F}$ & $\mathrm{D}$ & 0 & AVP & $\begin{array}{c}1 / 3 \\
\text { inférieur }\end{array}$ & Non & $\begin{array}{c}\text { Oui } \\
\text { Récupération } \\
\text { complète }\end{array}$ & 1 plaque & 4 & 0 & Non & 60 \\
\hline 12 & 62 & $\mathrm{~F}$ & G & 0 & Chute & $1 / 3$ moyen & Non & 0 & Orthopédique & 4 & 0 & Non & 18 \\
\hline 13 & 31 & $M$ & G & Oui & Chute & $1 / 3$ moyen & Non & $\begin{array}{c}\text { Oui } \\
\text { Récupération } \\
\text { complète }\end{array}$ & Clou & 4 & 0 & Non & 28 \\
\hline 14 & 53 & $M$ & D & Sevré & Chute & $1 / 3$ moyen & Non & 0 & 1 plaque & 1 & 0 & Non & 6 \\
\hline 15 & 59 & $\mathrm{M}$ & D & 0 & AVP & $\begin{array}{c}1 / 3 \\
\text { inférieur }\end{array}$ & Non & 0 & Clou & 3 & 0 & Non & 12 \\
\hline
\end{tabular}

\section{TABLEAU 1 : DESCRIPTION DES PATIENTS ET DES LESIONS}

(PR : Paralysie Radiale ; PDS : Perte de substance ; ATCD : antécédent ; PSA : Pseudarthrose ) 


\begin{tabular}{|l|c|c|c|c|c|}
\hline Auteur & Année & Greffe iliaque & \% consolidation & \% PR & \% infection \\
\hline Abalo (22) & 2011 & oui & 100 & 8 & 10 \\
\hline Barquet (23) & 1989 & oui & 96 & 4 & 0 \\
\hline Bernard (24) & 2010 & oui & 95 & 5 & 0 \\
\hline Hierholzer (25) & 2006 & oui & 99 & 8 & 0 \\
\hline Hsu (26) & 2005 & oui & 100 & 4 & 3 \\
\hline Kumar (27) & 2002 & oui & 91 & 5 & 10 \\
\hline Lin (28) & 2009 & oui & 100 & 4,6 & 3,4 \\
\hline Livani (29) & 2009 & non & 100 & 0 & 0 \\
\hline Singh (7) & 2014 & oui & 100 & 10 & 15 \\
\hline Wang (30) & 2013 & oui & 100 & 10 & 0 \\
\hline
\end{tabular}

TABLEAU 2 : PRINCIPALES SERIES EVALUANT LES TRAITEMENTS PAR PLAQUE DES PSA DE LA DIAPHYSE HUMERALE.

\begin{tabular}{|l|c|c|c|c|c|}
\hline Auteur & Année & Greffe iliaque & \% consolidation & $\%$ PR & \% infection \\
\hline Dujardin (31) & 2000 & non & 62 & 0 & 0 \\
\hline Flinkkila (32) & 2001 & non & 46 & 0 & 0 \\
\hline Ilyas (33) & 2003 & oui & 56 & 11 & 0 \\
\hline Li (34) & 2008 & oui & 100 & 0 & 0 \\
\hline Lin (8) & 2003 & oui & 96 & 2 & 0 \\
\hline Martinez (35) & 2002 & oui & 100 & 0 & 0 \\
\hline Singh (7) & 2014 & non & 85 & 0 & 10 \\
\hline Verbruggen (36) & 2005 & oui & 90 & 5 & 15 \\
\hline
\end{tabular}

TABLEAU 3 : PRINCIPALES SERIES EVALUANT LES TRAITEMENTS PAR CLOUS DES PSA DE LA DIAPHYSE HUMERALE.

\begin{tabular}{|l|c|c|c|c|c|}
\hline Auteur & Année & Greffe iliaque & \% consolidation & $\begin{array}{c}\text { \% lésions } \\
\text { nerveuses }\end{array}$ & $\%$ infections \\
\hline Bari (37) & 2004 & non & 100 & 0 & 0 \\
\hline Kiran (38) & 2010 & non & 100 & 5 & 9,8 \\
\hline Lammens (39) & 1998 & non & 93 & 10 & 5 \\
\hline Lavini (40) & 2001 & non & 100 & 0 & 20 \\
\hline Patel (41) & 2000 & non & 93 & 18 & 75 \\
\hline
\end{tabular}




\section{ICONOGRAPHIE}

FIGURE 1 - $1^{\text {ER }}$ TEMPS OPERATOIRE - NEUROLYSE DU NERF RADIAL ET ISOLEMENT DE SES RAMEAUX SENSITIVOMOTEURS

FIGURE $2: 1^{\text {ER }}$ TEMPS OPERATOIRE - TRANSPOSITION DU NERF AU TRAVERS DU FOYER DE PSEUDARTHROSE. OSTEOSYNTHESE PAR DOUBLE PLAQUE. ASPECT AVANT MISE EN PLACE D'UN ENGAINEMENT AU CIMENT

FIGURE $3: 2^{\text {EME }}$ TEMPS OPERATOIRE - OUVERTURE DE LA MEMBRANE

FIGURE $4: 2^{\text {EME }}$ TEMPS OPERATOIRE - ASPECT DE LA MEMBRANE APRES RETRAIT DU SPACER

FIGURE 5 : CAS 1 - PSEUDARTHROSE HUMERALE REBELLE DEJA OPEREE A 4 REPRISES.

FIGURE 6 : CAS 1 - ASPECT POST OPERATOIRE APRES L'APPORT OSSEUX. LE $1{ }^{\text {ER }}$ TEMPS AVAIT CONSISTE EN UNE REDUCTION ET SYNTHESE EN RACCOURCISSEMENT/COMPRESSION PAR 2 PLAQUES PUIS APPOSITION DES TUILES DE CIMENTS AU POURTOUR DU FOYER

FIGURE 7 : CAS 1 - ASPECT POST OPERATOIRE A 2 MOIS AVEC DEJA UN BEL ASPECT D'INTEGRATION DE LA GREFFE.

FIGURE 8 : CAS 1 - ASPECT POST OPERATOIRE A 6 MOIS. CONSOLIDATION ACQUISE

FIGURE 9 : CAS 2 - PSEUDARTHROSE HUMERALE REBELLE DEJA OPEREE A 6 REPRISES AYANT NECESSITE UNE COUVERTURE PAR LAMBEAU CHINOIS ; ASPECT AVANT $1^{\text {ER }}$ TEMPS

FIGURE 10 : CAS 3 - ASPECT POST OPERATOIRE APRES L'APPORT OSSEUX.

FIGURE 11 : CAS 2 - ARTHRODESE DE COUDE DE PAR LA DESTRUCTION EPIPHYSAIRE CHEZ UNE PATIENTE JEUNE. PONTAGE DE LA PERTE DE SUBSTANCE PAR UN GREFFON FIBULAIRE ASSOCIE A UN APPORT SPONGIEUX. CONSOLIDATION 


\section{ICONOGRAPHIE}

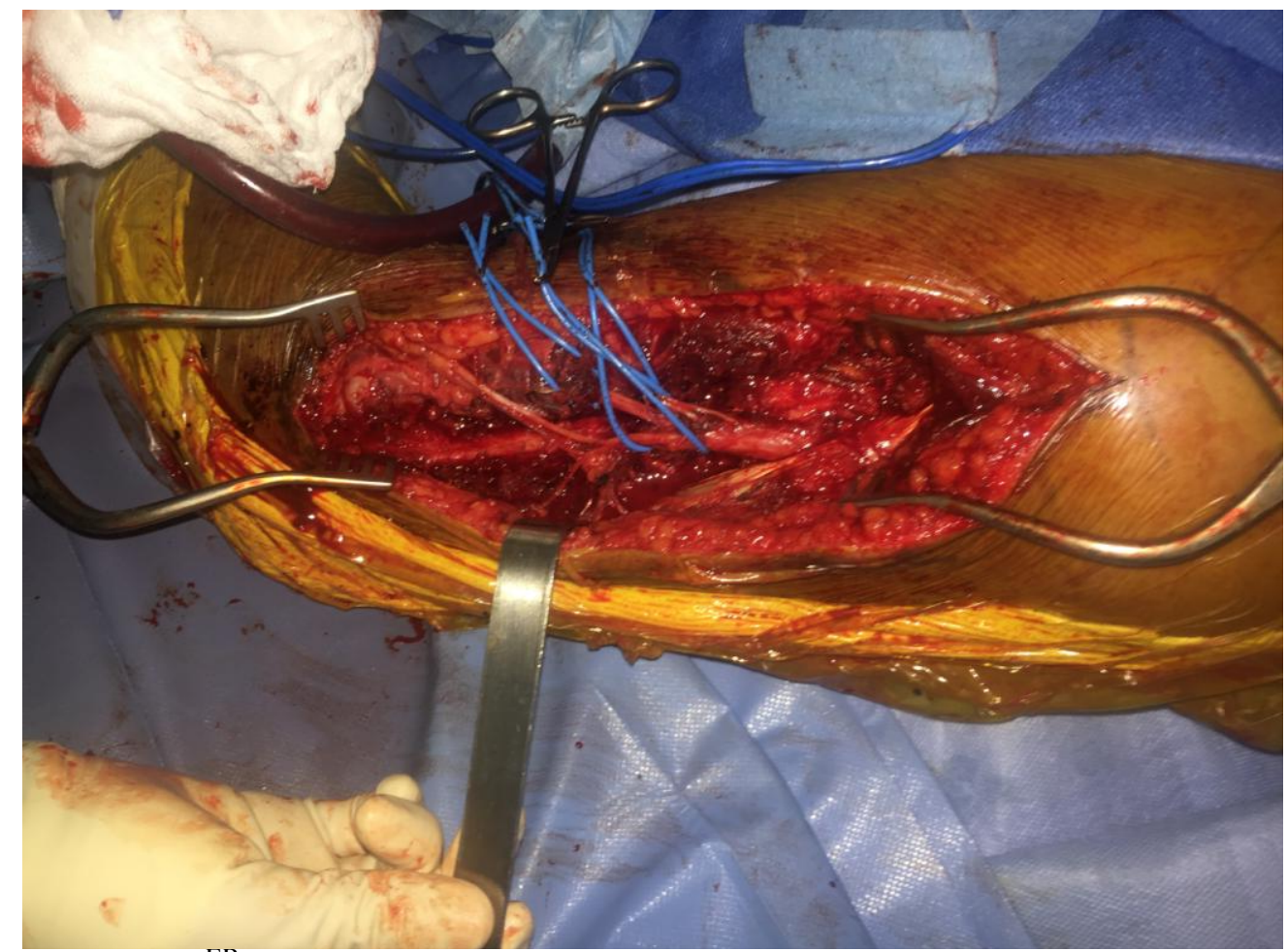

FIGURE $1: 1^{\text {ER }}$ TEMPS OPERATOIRE - NEUROLYSE DU NERF RADIAL ET ISOLEMENT DE SES RAMEAUX SENSITIVOMOTEURS

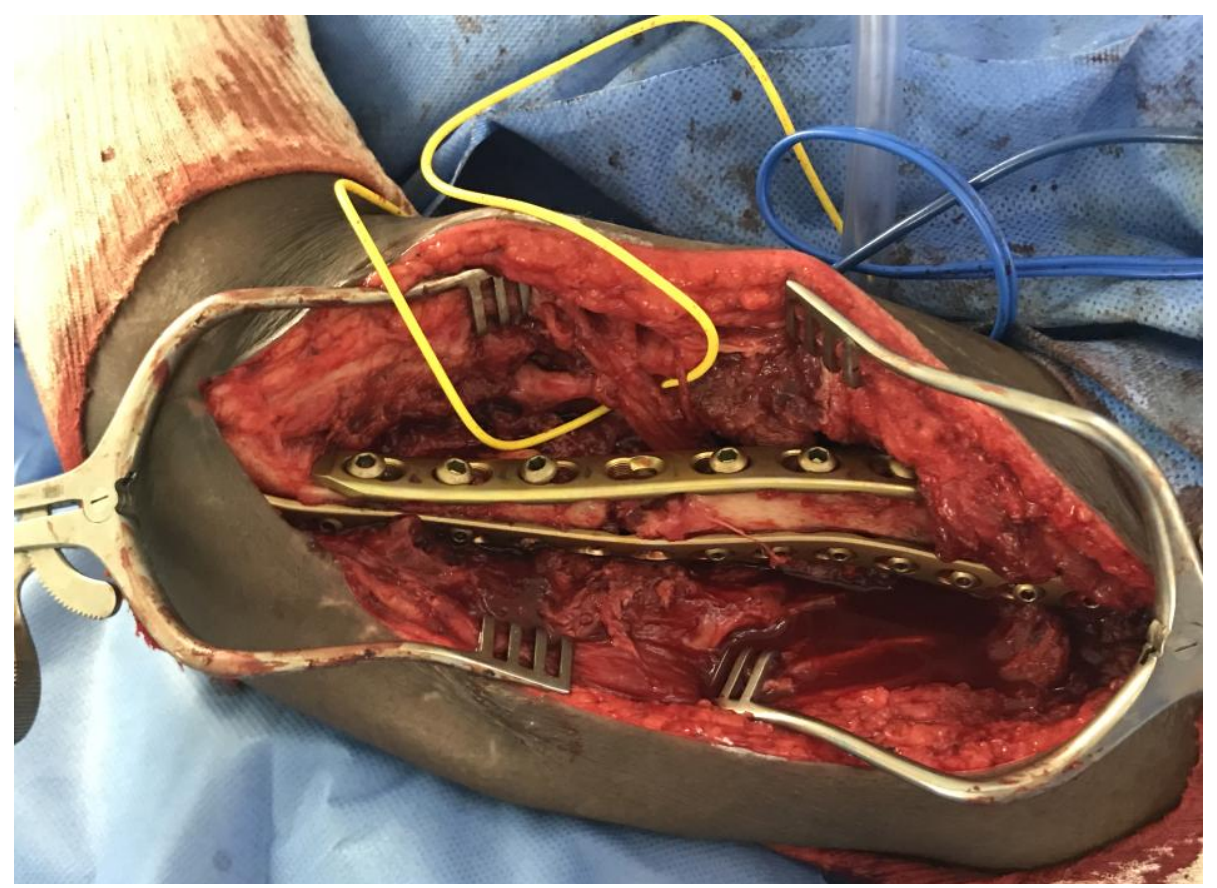

FIGURE $2: 1^{\text {ER }}$ TEMPS OPERATOIRE - TRANSPOSITION DU NERF AU TRAVERS DU FOYER DE PSEUDARTHROSE. OSTEOSYNTHESE PAR DOUBLE PLAQUE. ASPECT AVANT MISE EN PLACE D’UN ENGAINEMENT AU CIMENT 


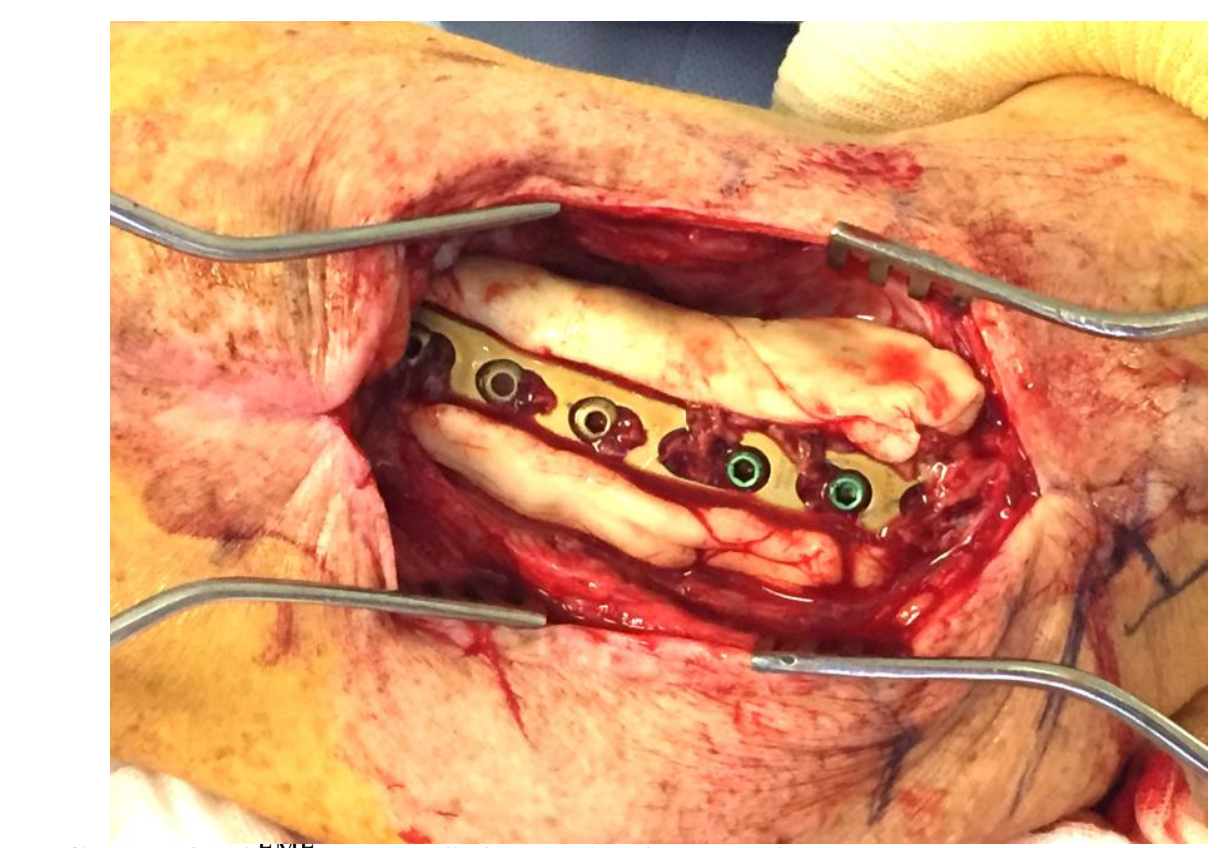

FIGURE $3: 2^{\text {EME }}$ TEMPS OPERATOIRE - OUVERTURE DE LA MEMBRANE

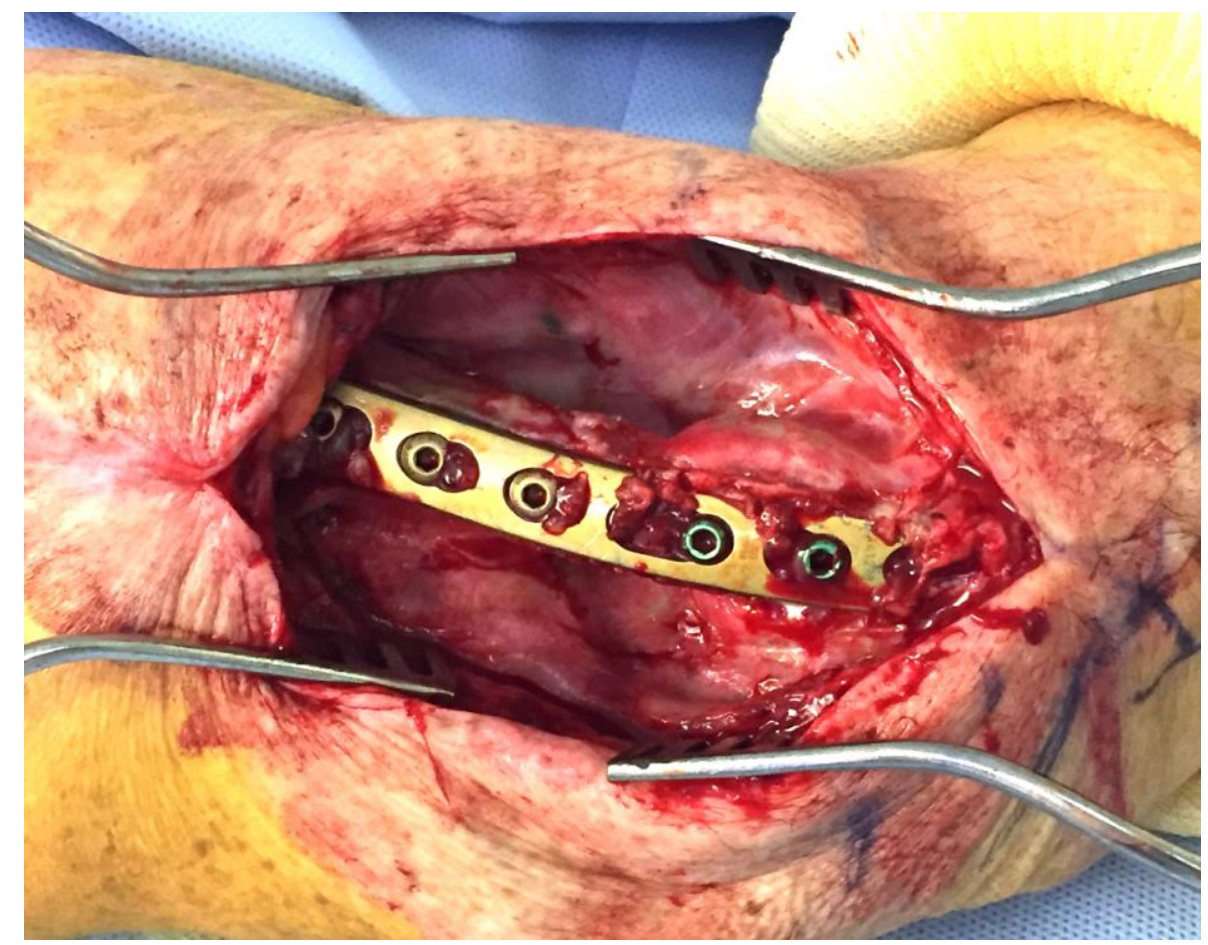

FIGURE $4: 2^{\text {EME }}$ TEMPS OPERATOIRE - ASPECT DE LA MEMBRANE APRES RETRAIT DU SPACER 


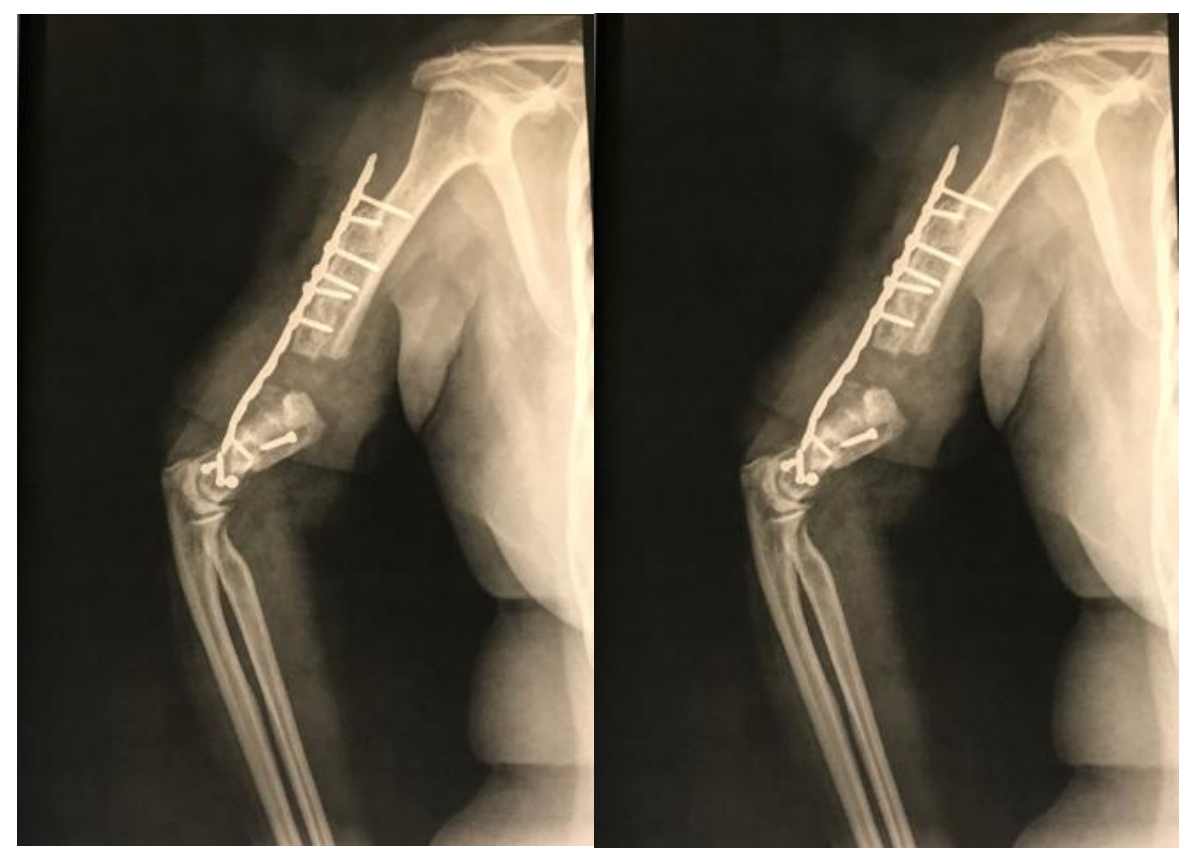

FIGURE 5 : CAS 1 - PSEUDARTHROSE HUMERALE REBELLE DEJA OPEREE A 4 REPRISES.

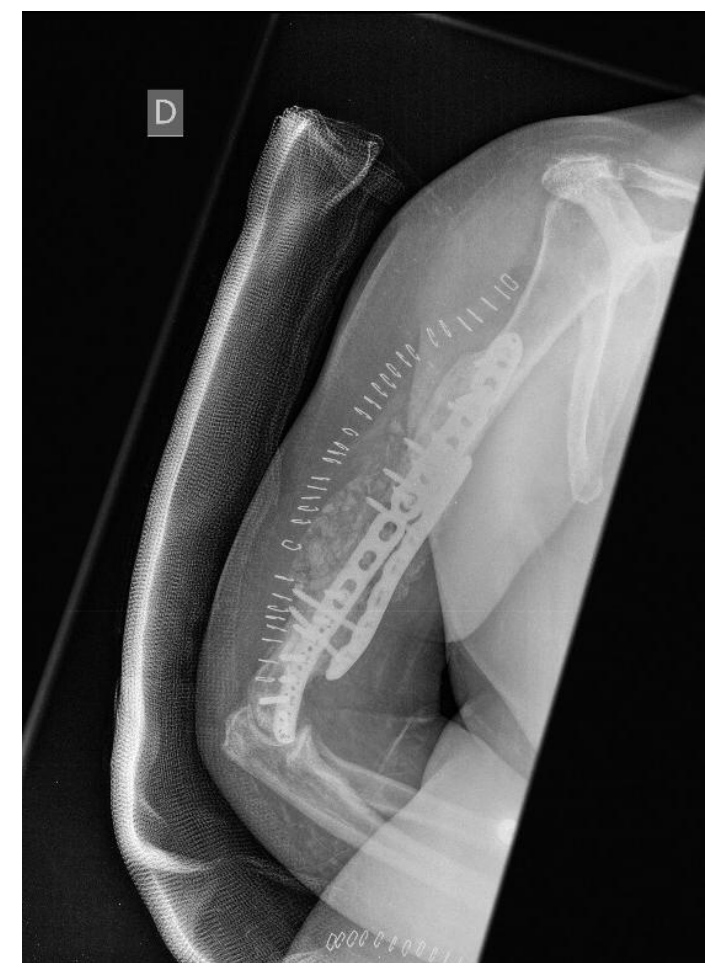

FIGURE 6 : CAS 1 - ASPECT POST OPERATOIRE APRES L'APPORT OSSEUX. LE $1^{\text {ER }}$ TEMPS AVAIT CONSISTE EN UNE REDUCTION ET SYNTHESE EN RACCOURCISSEMENT/COMPRESSION PAR 2 PLAQUES PUIS APPOSITION DES TUILES DE CIMENTS AU POURTOUR DU FOYER 


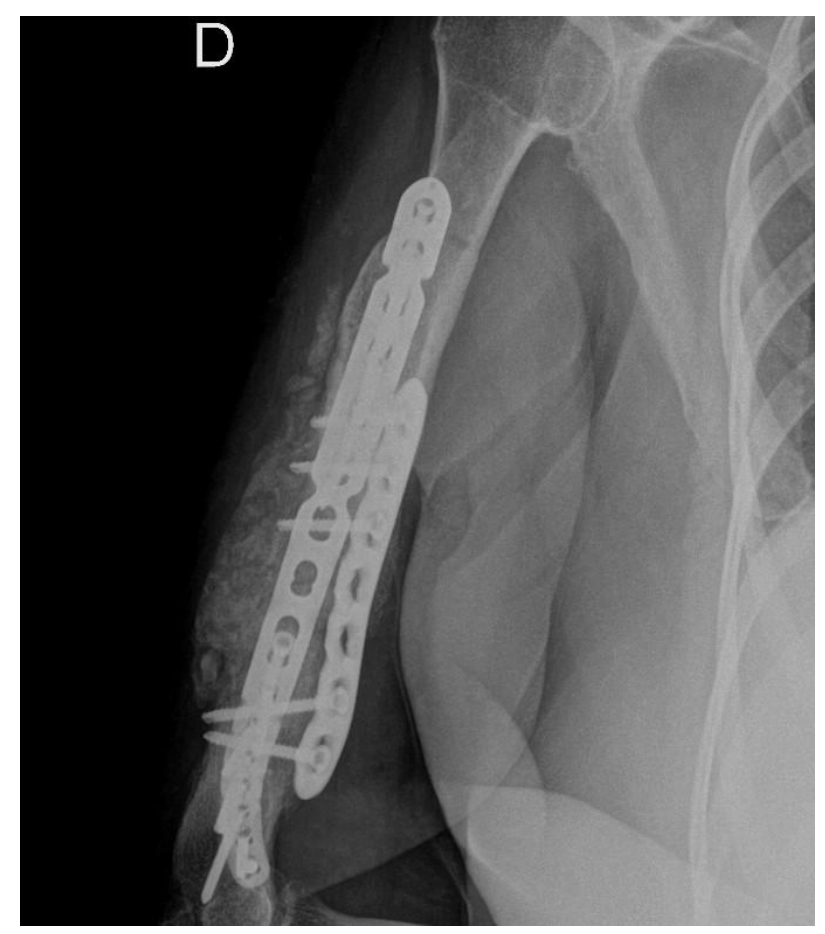

FIGURE 7 : CAS 1 - ASPECT POST OPERATOIRE A 2 MOIS AVEC DEJA UN BEL ASPECT D'INTEGRATION DE LA GREFFE.
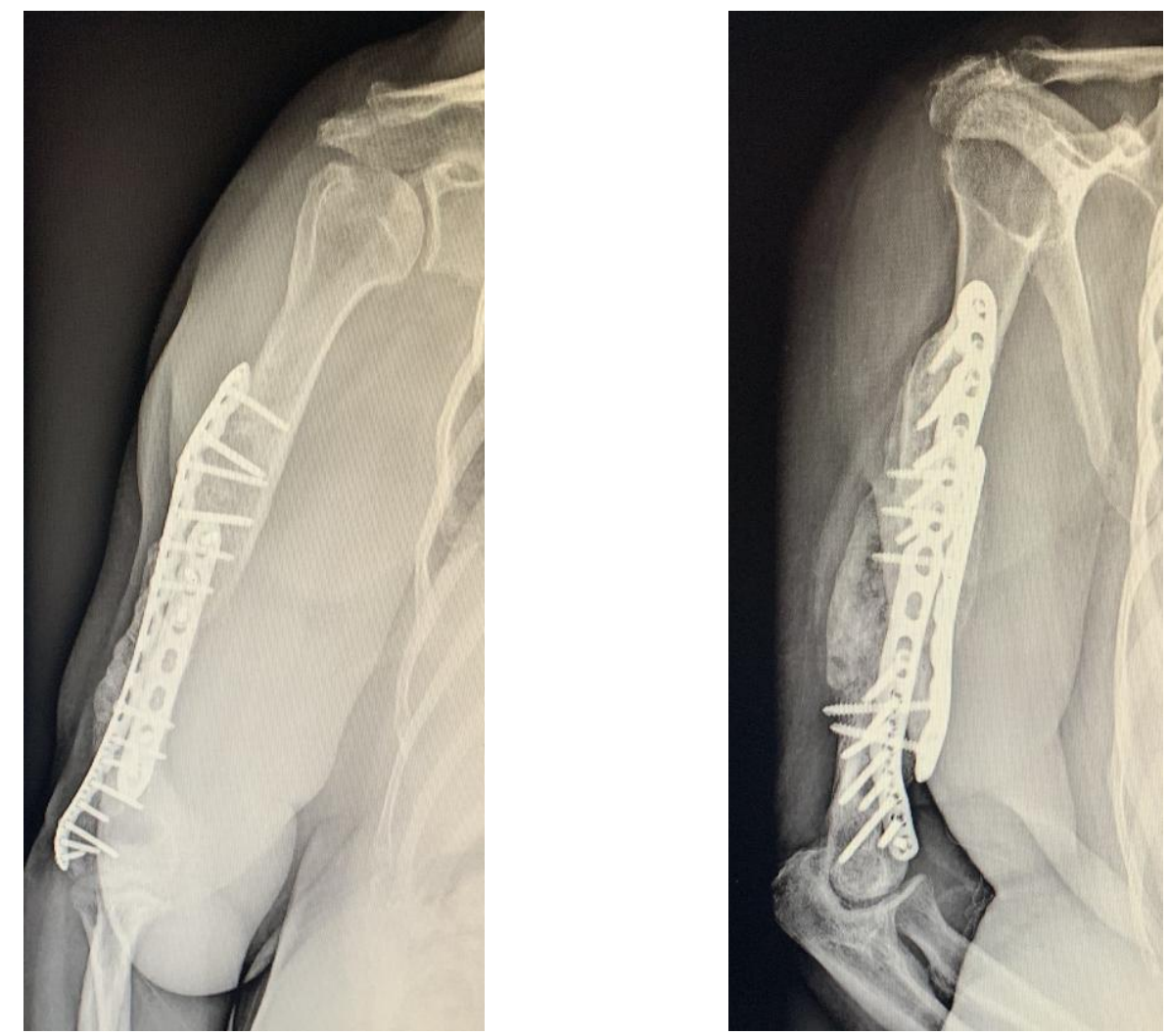

FIGURE 8 :

CAS 1 - ASPECT POST OPERATOIRE A 6 MOIS. CONSOLIDATION ACQUISE 


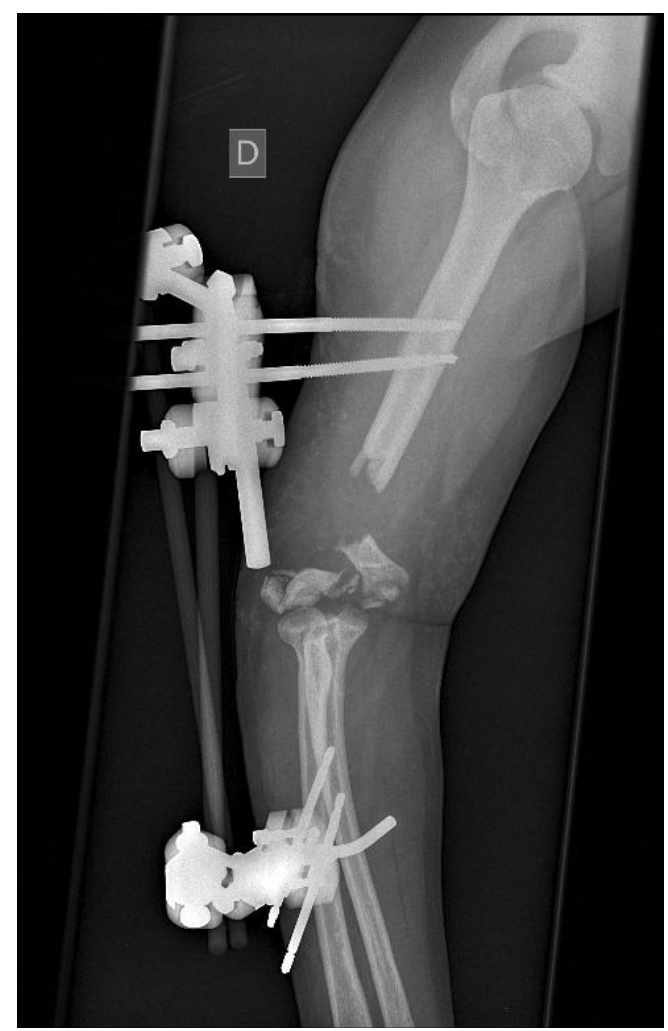

FIGURE 9 : CAS 2 - PSEUDARTHROSE HUMERALE REBELLE DEJA OPEREE A 6 REPRISES AYANT NECESSITE UNE COUVERTURE PAR LAMBEAU CHINOIS ; ASPECT AVANT $1^{\text {ER }}$ TEMPS

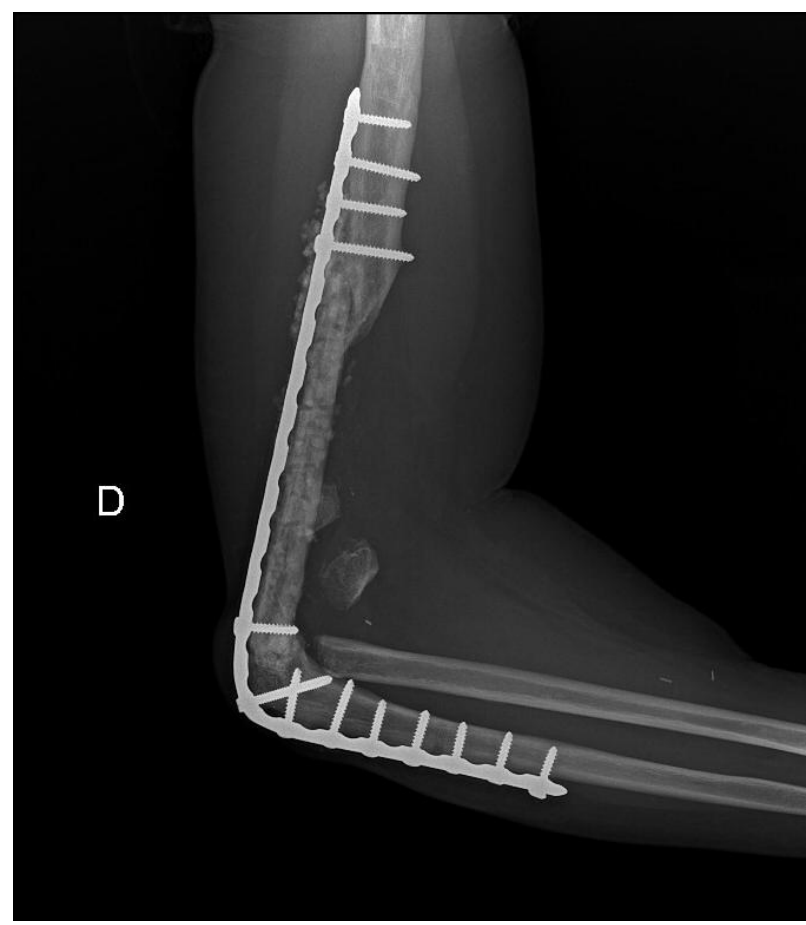

FIGURE 10 : CAS 2 - ARTHRODESE DE COUDE DE PAR LA DESTRUCTION EPIPHYSAIRE CHEZ UNE PATIENTE JEUNE. PONTAGE DE LA PERTE DE SUBSTANCE PAR UN GREFFON FIBULAIRE ASSOCIE A UN APPORT SPONGIEUX. CONSOLIDATION 